\title{
Trans-Planckian Physics from a Nonlinear Dispersion Relation
}

\author{
S.E. Jorás \\ Instituto de Física, Universidade Federal do Rio de Janeiro, \\ Caixa Postal 68528, Rio de Janeiro, RJ 21941-972, Brazil \\ G. Marozzi \\ Dipartimento di Fisica, Università degli Studi di Bologna and INFN, via Irnerio 46, I-40126 Bologna, Italy
}

\begin{abstract}
We study a particular nonlinear dispersion relation $\omega_{p}\left(k_{p}\right)$ - a series expansion in the physical wavenumber $k_{p}$ - for modeling first-order corrections in the equation of motion of a test scalar field in a de Sitter spacetime from trans-Planckian physics in cosmology. Using both a numerical approach and a semianalytical one, we show that the WKB approximation previously adopted in the literature should be used with caution, since it holds only when the comoving wavenumber $k \gg a H$. We determine the amplitude and behavior of the corrections on the power spectrum for this test field. Furthermore, we consider also a more realistic model of inflation, the power-law model, using only a numerical approach to determine the corrections on the power spectrum.
\end{abstract}

\section{INTRODUCTION}

Inflationary models provide answers to many problems in standard big bang cosmology, in particular the origin of density fluctuations and the spectrum of cosmic microwave background anisotropies. The basis of the whole mechanism is the stretching of quantum fluctuations generated at sub-Hubble scales due to the exponential expansion of the spacetime during inflation. This model, however, has a serious "problem": if we consider a plain scalar-field-driven inflationary model - say, chaotic inflation - the period of inflation lasts so long that the wavelengths of the fluctuations which at present correspond to cosmological scales were sub-Planckian at the begining of the inflationary phase. Therefore, the evolution of fluctuations at such scales is supposed to follow different rules from those provided by the standard theory of cosmological perturbations - which is based on quantum field theory and general relativity. The set of rules expected to hold above this energy scale is the socalled trans-Planckian physics (TPP from now on). The TPP could lead to deviations from standard predictions on the cosmic microwave background radiation (CMBR), which probes the scales we mentioned before. The question to be asked is "whether the predictions of the standard cosmology are insensitive to effects of TPP". This is the precise statement of the trans-Planckian problem [1, 2].

In the present paper we adopt, to mimic TPP, a seriesexpansion expression for the nonlinear dispersion relation $\omega_{p}$ (Eq. (19) below), first suggested by [3], to modify the equation of motion of a test scalar field in a de Sitter spacetime. We argue that, in this framework, the WKB approximation is valid only for $k \gg a H$ (or $k_{p} \equiv k / a \gg$ $H$ with $k_{p}$ the physical wavenumber and $H$ the Hubble factor). As a consequence, the perturbative approach based on the WKB approximation, used in [4] to tackle this issue, should be used with caution.

The outline of the paper is the following. In Sec. I we present the standard approach for first-order cosmolog- ical perturbations and then we restrict ourselves to the case of a test scalar field in a de Sitter spacetime. We also introduce the WKB approximation in this section. In Sec. [II] we adopt a particular way to approach TPP: a modification of the equation of motion due to a nonlinear dispersion relation. The initial conditions for the test scalar field are set in Sec. IV] In the following sections we present a numerical calculation, a 3-piecewise approximation (adopted in Ref. [4]) and a semianalytical approach to solve the problem. In Sec. VIII] we investigate a realistic model of inflation, namely power-law inflation, using the same nonlinear dispersion relation. We then conclude in Sec. IX,

\section{STANDARD APPROACH}

The spatially flat Friedmann-Robertson-Walker (FRW) metric with first-order cosmological fluctuations is given by $\left(d s^{2}=g_{\mu \nu} d x^{\mu} d x^{\nu}\right)$ :

$$
\begin{aligned}
g_{00}= & a^{2}(-1-2 \alpha) \\
g_{0 i}= & -\frac{a^{2}}{2}\left(\beta_{, i}+B_{i}\right) \\
g_{i j}= & a^{2}\left[\delta_{i j}(1-2 \psi)+D_{i j} E+\right. \\
& \left.+\left(\chi_{j, i}+\chi_{i, j}+h_{i j}\right) / 2\right] .
\end{aligned}
$$

with $D_{i j}=\partial_{i} \partial_{j}-1 / 3 \nabla^{2} \delta_{i j}$, considering the conformal time $\eta$. To first-order scalar, vector and tensor perturbations evolve independently. Vector perturbations can be omitted because they die away kinematically. The tensor perturbation $h_{i j}$ has only two physical degrees of freedom (polarization states) $h_{+}$and $h_{*}$ :

$$
h_{i j}=h_{+} e_{i j}^{+}+h_{*} e_{i j}^{*}
$$

where $e_{i j}^{+}$and $e_{i j}^{*}$ are the polarization tensors having the following properties in the Fourier space:

$$
e_{i j}=e_{j i}, \quad k^{i} e_{i j}=0, \quad e_{i}^{i}=0
$$




$$
e_{i j}^{+} e^{+i j}=2, \quad e_{i j}^{*} e^{* i j}=2, \quad e_{i j}^{+} e^{* i j}=0
$$

On expanding in Fourier modes we can define

$$
h_{\lambda}(t, \vec{x})=\frac{1}{a(\eta)} \frac{1}{(2 \pi)^{3 / 2}} \int d^{3} k \mu_{t k}(\eta) e^{i \vec{k} \cdot \vec{x}},
$$

with $\lambda=+/ *$ and where $\mu_{t k}$ will satisfy [5]:

$$
\mu_{t k}^{\prime \prime}+\left(k^{2}-\frac{a^{\prime \prime}}{a}\right) \mu_{t k}=0
$$

The scalar sector, in the case of a plain scalar field $\phi(\eta, \vec{x})=\phi(\eta)+\varphi(t, \vec{x})$ driven inflationary model, can be reduced to the study of a single gauge-invariant scalar variable defined by

$$
Q=\frac{\mu_{s}}{a}=\varphi+\frac{\phi^{\prime}}{\mathcal{H}}\left(\psi+\frac{1}{6} \nabla^{2} E\right)
$$

where $\mathcal{H}=\frac{a^{\prime}}{a}$. This so-called Mukhanov variable [6] obeys the following equation of motion in Fourier space:

$$
\mu_{s k}^{\prime \prime}+\left(k^{2}-\frac{z^{\prime \prime}}{z}\right) \mu_{s k}=0
$$

with $z=a \frac{\phi^{\prime}}{\mathcal{H}}$. Considering an adiabatic vacuum state as initial condition for those perturbations, all the statistical properties are characterized by the two-point correlation function, namely by the power spectrum. The dimensionless power spectrum for scalar and tensor cosmological fluctuations are given, respectively, by

$$
P_{Q}=\frac{k^{3}}{2 \pi^{2}}\left|\frac{\mu_{s k}}{z}\right|^{2} \quad, \quad P_{h}=\frac{2 k^{3}}{\pi^{2}}\left|\frac{\mu_{t k}}{a}\right|^{2} .
$$

Their dependence on the mode $k$ is defined by the spectral index in the following way:

$$
n_{s}-1 \equiv \frac{d \ln P_{Q}}{d \ln k} \quad, \quad n_{t} \equiv \frac{d \ln P_{h}}{d \ln k}
$$

evaluated at a scale $k \ll a H$ when the mode is outside the horizon. For $n_{s}=1$ one has a scale-invariant spectrum for the gauge-invariant cosmological scalar fluctuation.

Let us now restrict ourselves to the case of a test scalar field in a de Sitter spacetime where $a(\eta)=-1 /(H \eta)$. In this case, cosmological fluctuations vanish identicaly.

If we expand the test scalar field $\phi(\eta, \vec{x})$ in Fourier modes

$$
\phi(\eta, \vec{x})=\frac{1}{a(\eta)} \frac{1}{(2 \pi)^{3 / 2}} \int d^{3} k \mu_{k}(\eta) e^{i \vec{k} \cdot \vec{x}}
$$

then the equation of motion for each mode is given by

$$
\mu_{k}^{\prime \prime}+\Omega^{2}(\eta) \mu_{k}=0
$$

with

$$
\Omega^{2}(\eta) \equiv k^{2}-\frac{a^{\prime \prime}}{a}
$$

which becomes for a de Sitter spacetime

$$
\Omega^{2}(\eta)=k^{2}-\frac{2}{\eta^{2}} .
$$

We can note that Eq.(12) is the same equation of motion as that of a tensor perturbation (6). In this simple case, Eq. (12) can be exactly solved.

The two-point correlation function is given by

$$
\langle 0|\phi(\eta, \vec{x}) \phi(\eta, \vec{x}+\vec{r})| 0\rangle=\int_{0}^{+\infty} \frac{d k}{k} \frac{\sin k r}{k r} P_{\phi}(k)
$$

and the power spectrum is

$$
P_{\phi}(k)=\frac{k^{3}}{2 \pi^{2}}\left|\frac{\mu_{k}}{a}\right|^{2} .
$$

For superhorizon modes $(k \ll a H)$ the spectrum is time independent and scale invariant, as one can see from the exact solution in the limit $\eta \rightarrow 0^{-}$.

Equation (12) can also be interpreted as a Schrödinger equation for a stationary wave function with energy $E \equiv \omega^{2}=k^{2}$ in an effective potential $V_{\text {eff }}(\eta) \equiv a^{\prime \prime} / a$ which is a function of the "spatial" variable $\eta$. We might be tempted to solve this equation using WKB approximation, just as it is usually done in basic quantum mechanics (QM) 7]. In this approximation the stationary solution of Eq. (12) is given by

$$
\begin{aligned}
\mu_{k}(\eta) & =\frac{c_{+}}{\Omega^{1 / 2}} \exp \left[+i \int^{\eta} \Omega\left(\eta^{\prime}\right) d \eta^{\prime}\right]+ \\
& +\frac{c_{-}}{\Omega^{1 / 2}} \exp \left[-i \int^{\eta} \Omega\left(\eta^{\prime}\right) d \eta^{\prime}\right]
\end{aligned}
$$

as long as the WKB parameter $W$ is much smaller than 1:

$$
W \equiv\left|\frac{1}{\Omega^{2}}\left[\frac{3}{4}\left(\frac{\Omega^{\prime}}{\Omega}\right)^{2}-\frac{1}{2} \frac{\Omega^{\prime \prime}}{\Omega}\right]\right| \ll 1 \quad .
$$

Figure 1 shows the behavior of $W$ as a function of the conformal time $\eta$ in a de Sitter case. According to this plot, the WKB approximation holds for $\eta \rightarrow-\infty$ ( $k \gg a H$, subhorizon scales) but not for values close to zero ( $k \ll a H$, superhorizon scales), where it equals 0.125 (i.e, not much smaller than 1) [8]. It fails exactly where it is supposed to: near the classical turning point $\left(\eta_{\mathrm{tp}} \equiv\right.$ $-\sqrt{2} / k)$ and where the effective potential is too steep $\left(\eta \rightarrow 0^{-}\right)$. For the subhorizon scales, where $E \gg V_{\text {eff }}$, the solution of Eqs. (1214) is a plane wave in conformal time with comoving frequency $\Omega \simeq k$, Eq. (17), as expected.

\section{TRANS-PLANCKIAN BEHAVIOR: NON-LINEAR DISPERSION RELATION}

A modification of the linear dispersion relation (d.r. from now on) was proposed by Unruh [9] for describing 


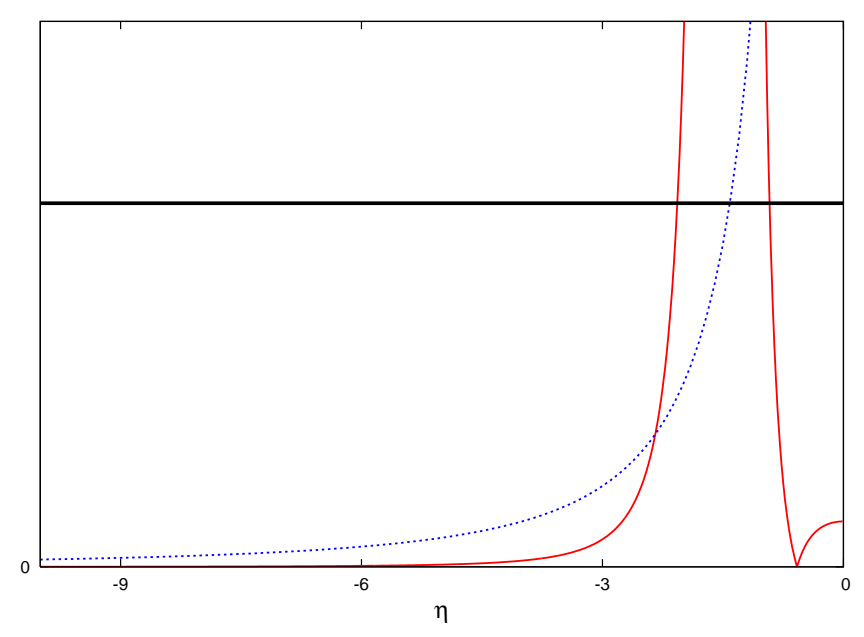

FIG. 1: Total energy (full straight horizontal line), effective potential (dashed blue curve), and WKB parameter (full red curve) as a function of the conformal time $\eta$ for a linear dispersion relation. We have used $k=M_{p l}$ and $\eta$ is in units of $1 / M_{p l}$.

high-energy Physics in the black-hole radiation emission. He was inspired by sound waves, for which a linear d.r. ceases to be valid when the wavelength gets closer to or smaller than the lattice spacing. Jacobson and Corley [10] (see also [2]) also proposed nonlinear terms in the d.r. that could be justified by the inclusion of higher-order derivatives in the Lagrangian. Other changes can also be introduced by arguing that the spacetime symmetries migh not survive at high energies [11].

Following this approach to mimic TPP one writes the comoving frequency $\omega$ as $\omega=a(\eta) \omega_{p}\left(k_{p}\right)$ - where $\omega_{p}$ is the physical frequency - and assumes that $\omega_{p}$ is a nonlinear function of $k_{p}$ which differs from the standard (linear) one only for physical wavelengths closer to or smaller than the Planck scale. Note that this replacement would be innocuous if the dispersion relation was linear in $k_{p}$.

In this paper we focus on the d.r.

$$
\omega_{p}^{2}\left(k_{p}\right)=k_{p}^{2}-\alpha k_{p}^{4}+\beta k_{p}^{6},
$$

with $\alpha>0$ and $\beta>0$, proposed in Ref. [3]. See Fig. 2 for a sketch of this function. The above expression can be seen as a mere series expansion, but it is also found in solid state physics and describes the rotons [12]. There has been suggestions [13] for including a 3-order term in the nonlinear d.r. above, coming from an effective-fieldtheory approach. Since such odd-power terms violate CP, we do not consider them here.

Following the discussion in Section II, we plot in Fig. 3 the analogous quantities to $W$ (18), the total energy $E \equiv$ $k^{2}$, and the effective potential, the latter being given by

$$
V_{\mathrm{eff}}(\eta) \equiv\left(\bar{\alpha} k^{4}\right) \eta^{2}-\left(\bar{\beta} k^{6}\right) \eta^{4}+\frac{2}{\eta^{2}}
$$

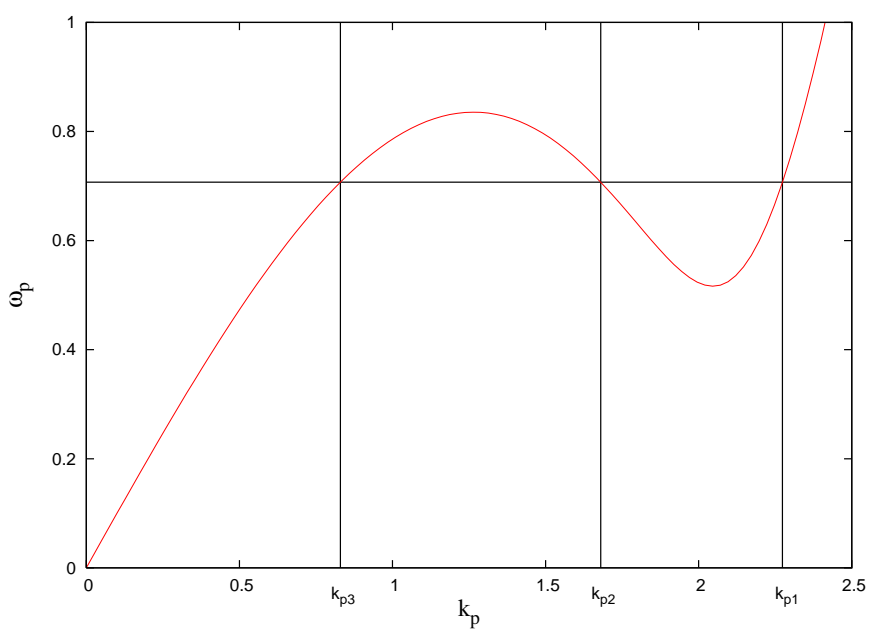

FIG. 2: Nonlinear dispersion relation as a function of the physical wavenumber $k_{p}$ for a case with three different solutions $\left(k_{p 1}, k_{p 2}\right.$ and $\left.k_{p 3}\right)$ of the turning-point equation $\omega_{p}^{2}=$ $2 H^{2} . H=0.5 M_{p l}$ and $k_{p}$ is in units of $M_{p l}$.

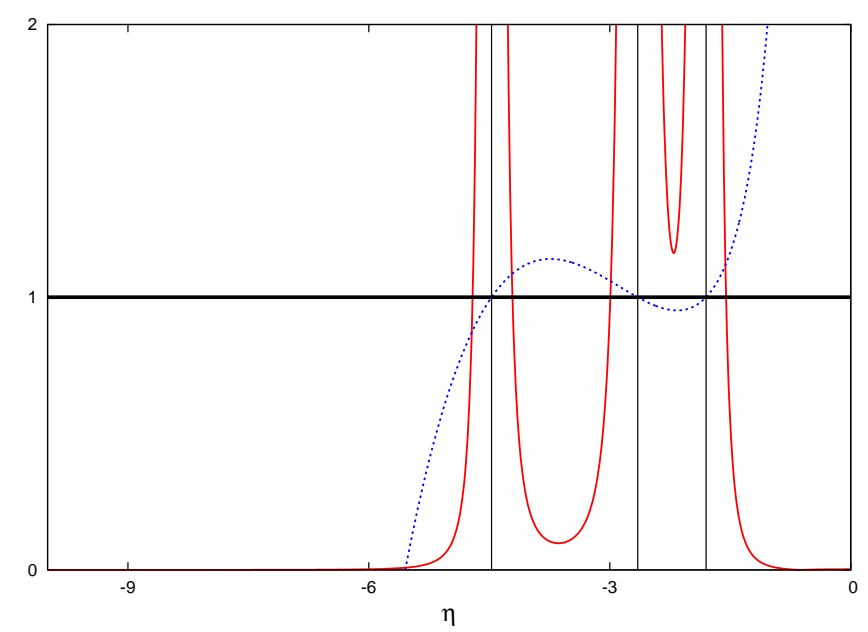

FIG. 3: Total energy (full straight horizontal line), effective potential (dashed blu curve), and WKB parameter (full red curve) as a function of the conformal time $\eta$ for the nonlinear dispersion relation Eq. (19). We have used $\bar{\alpha}=0.1325$, $\bar{\beta}=0.004375$ and $H=0.5 M_{p l}$ so that there are 3 turning points (indicated by the vertical lines). The WKB curve was multiplied by $2 \cdot 10^{-2}$ so its behavior in the region close to the turning points can be clearly seen. Note, however, that the WKB approximation is valid only in the region $\eta \rightarrow-\infty$ since $W\left(\eta \rightarrow 0^{-}\right)=0.125$. We have used $k=M_{p l}$ and $\eta$ is in units of $1 / M_{p l}$.

where $\bar{\alpha} \equiv \alpha H^{2}$ and $\bar{\beta} \equiv \beta H^{4}$ are dimensionless quantities. As in the linear case, the WKB approximation is valid only when $\eta \rightarrow-\infty$.

To compare our results with Ref. [4] we consider only a particular range of values for $\alpha$ and $\beta$, necessary for a positive nonlinear dispersion relation with three real distinct solutions for the classical turning-points equation $\omega_{p}^{2}=2 H^{2}$ (see, also, [14] ). The request to have three real 
turning-points and a nonlinear $\omega_{p}$ that differs from the standard linear one only for physical wavelengths closer to or smaller than the Planck scale has as consequence that $H$ should be comparable with $M_{p l}$. In fact, everywhere in the paper we consider $H=0.5 M_{p l}$.

Defining $z \equiv 3 \bar{\beta} / \bar{\alpha}^{2}$ one obtains the following ranges for $z$ and $\bar{\alpha}$ :

$$
3 / 4<z<1 \quad, \quad g(z)<\bar{\alpha}<f(z)
$$

with

$$
\begin{aligned}
& g(z) \equiv \frac{1}{3 z^{2}}\left[\frac{3 z}{2}-1-(1-z)^{3 / 2}\right] \\
& f(z) \equiv \frac{1}{3 z^{2}}\left[\frac{3 z}{2}-1+(1-z)^{3 / 2}\right]
\end{aligned}
$$

where $z>3 / 4$ is the physical condition to have a positive nonlinear d.r.. The problem of finding the power spectrum from this particular nonlinear dispersion relation has been tackled in Ref. [4]. In that paper the authors introduced an approximate piecewise form of the nonlinear dispersion relation (see Sec VI) and apply the WKB approximation for $k_{p}$ such that $\omega_{p}^{2}>2 H^{2}$. They argue that the amplitude of the effects of the nonlinearity is proportional to $\Delta \equiv\left(k_{p 1}-k_{p 2}\right) / k_{p 1}$ where $k_{p 1}$ and $k_{p 2}$ are, respectively, the first and the second turning points from the right of Fig. 2, Still according to Ref. [4], $\Delta$ measures the "time" spent in the region where the WKB approximation is not satisfied, but it actually is simply a measure of the distance between the two largest turning points $-k_{p 1}$ and $k_{p 2}$ in Fig. 2, It is clearly seen in Fig. 3 that $\Delta$ is much smaller that such time interval.

We show below the full result (i.e, the exact solution within the three-piecewise approximation) and two other different approaches - one numerical and the other semianalytical - for evaluating the nonlinear effects on the power spectrum.

But before proceeding to proving the claims above, we shall first determine the initial conditions we assume.

\section{THE EQUATION OF MOTION: INITIAL CONDITIONS}

The equation of motion for the mode function of a test scalar field, following the approach just introduced to consider the TPP effects, is given by

$$
\mu_{k}^{\prime \prime}+\left(a^{2} \omega_{p}^{2}-\frac{a^{\prime \prime}}{a}\right) \mu_{k}=0
$$

with $\omega_{p}$ given by Eq.(19).

For $k \gg a H$ the WKB approximation is valid (see Fig. 3), the term $a^{2} \omega_{p}^{2}=\omega^{2}$ dominates and the mode function is given, chosing the adiabatic vacuum (see [4]), by:

$$
\mu_{w k b}(k, \eta)=\frac{1}{\sqrt{2 \omega(k, \eta)}} \exp \left[-i \int_{\eta_{i}}^{\eta} \omega(k, \tau) d \tau\right] .
$$

To proceed with either a numerical or an analytical approach to find the solution of Eq.(24) we have to find the initial conditions associated to our nonlinear dispersion relation in a de Sitter spacetime. We consider a initial time $\eta_{i}$ and a fixed value of $k$ (which should be much larger than $\left.a\left(\eta_{i}\right) H\right)$.

In this case, for this fixed value $k \gg a H$, the comoving frequency $\omega$ becomes nearly equal to $\sqrt{\beta} k^{3} / a^{2}$ and the WKB vacuum is given by

$$
\begin{aligned}
\mu_{w k b}(\eta)= & -\frac{1}{\sqrt{2} \beta^{1 / 4} k^{3 / 2}} \frac{1}{H \eta} \times \\
& \times \exp \left[-i \beta^{1 / 2} k^{3} \frac{H^{2}}{3}\left(\eta^{3}-\eta_{i}^{3}\right)\right],
\end{aligned}
$$

its derivative by

$$
\begin{aligned}
\mu_{w k b}^{\prime}(\eta)= & \left\{\frac{1}{\sqrt{2} \beta^{1 / 4} k^{3 / 2}} \frac{1}{H \eta^{2}}+i \frac{1}{\sqrt{2}} \beta^{1 / 4} k^{3 / 2} H \eta\right\} \times \\
& \times \exp \left[-i \beta^{1 / 2} k^{3} \frac{H^{2}}{3}\left(\eta^{3}-\eta_{i}^{3}\right)\right]
\end{aligned}
$$

and our initial conditions by

$$
\begin{aligned}
\mu_{w k b}\left(\eta_{i}\right) & =-\frac{1}{\sqrt{2} \beta^{1 / 4} k^{3 / 2}} \frac{1}{H \eta_{i}} \\
\mu_{w k b}^{\prime}\left(\eta_{i}\right) & =\frac{1}{\sqrt{2} \beta^{1 / 4} k^{3 / 2}} \frac{1}{H \eta_{i}^{2}}+i \frac{\beta^{1 / 4} k^{3 / 2}}{\sqrt{2}} H \eta_{i}
\end{aligned}
$$

To be more accurate the above equations are meaningful only when

$$
\Omega^{2}(\eta) \equiv k^{2}-\alpha \frac{k^{4}}{a^{2}}+\beta \frac{k^{6}}{a^{4}}-\frac{2}{\eta^{2}} \simeq \beta \frac{k^{6}}{a^{4}}
$$

so one obtains the following constraints on $\beta$ :

$$
\beta \gg 2 H^{2} \frac{a^{6}}{k^{6}} \quad, \quad \beta \gg \frac{a^{4}}{k^{4}} \quad, \quad \beta \gg \alpha \frac{a^{2}}{k^{2}} .
$$

The WKB solution is an exact one at the infinite past and thus the choice of the adiabatic vacuum is somewhat "natural". We also recall that all vacua prescriptions are equivalent up to zero-order when the WKB approximation holds [15].

The choice of a different set of initial conditions at a given $\eta=\eta_{1}$, though, can always be seen as the outcome from a particular (trans-Planckian) evolution from a different (and perhaps more consensual) set of initial conditions defined at $\eta=\eta_{0}<\eta_{1}$. In other words, the choice of initial conditions is equivalent to the choice of the physics that takes place before the moment when they are set. Nevertheless, that choice does not replace the discussion on the physics that takes place after that moment, while energies above the Planck scale are still at play. 


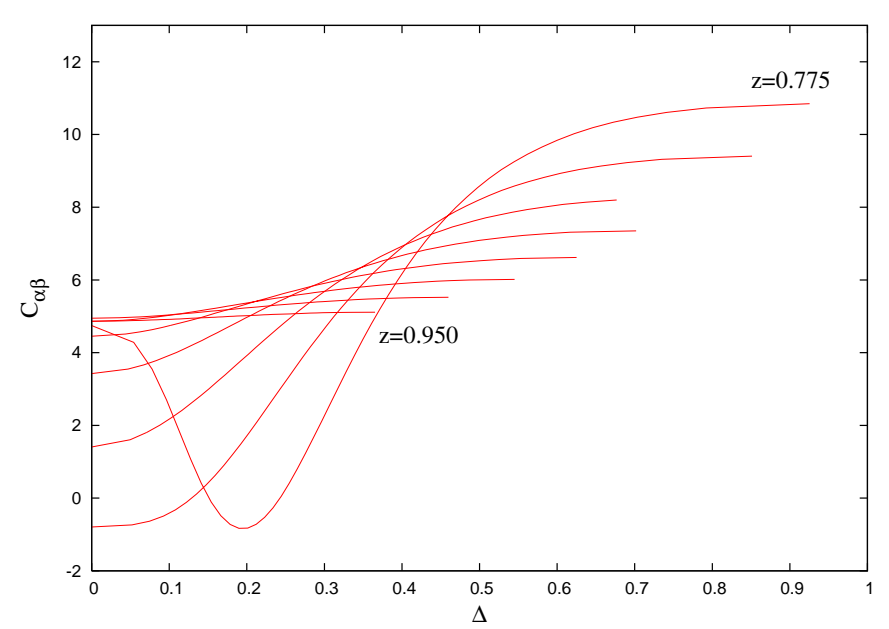

FIG. 4: Correction $C_{\alpha \beta}$ on the power spectrum, with $z$ varying from 0.775 up to 0.950 with step 0.025 , as a function of $\Delta$ from a full numerical calculation using the nonlinear d.r. (19).

\section{NUMERICAL APPROACH}

In this section we find the correction to the power spectrum, for our test scalar field in a de Sitter spacetime with a nonlinear d.r., using a fully numerical approach with initial conditions given by Eqs. (2829). This has been done using a $\mathrm{C}$ code and the GSL library [16], where we have set $H=0.5 M_{p l}$ and considered a fixed value of $k \gg a\left(\eta_{i}\right) H$, stopping the evolution at a time $\eta_{f}$ for which $k \ll a\left(\eta_{f}\right) H$. We have verified that the result is independent from the value of $k$, namely that, as expected, the power spectrum is still scale independent.

We write the power spectrum as

$$
P_{\phi}(k)=\left(\frac{H}{2 \pi}\right)^{2}\left[1+C_{\alpha \beta}\right] .
$$

The function $C_{\alpha \beta}$ represents the correction due to the nonlinear d.r., which obviously depends on the parameters $\alpha$ and $\beta$.

In Fig. 4 we show the correction $C_{\alpha \beta}$ for different values of $z$ (going from $z=0.775$ to 0.950 with step 0.025 ) in function of $\Delta$. We can clearly see that $\Delta$ is not the only parameter that plays a role in the calculation of the nonlinear effects on the power spectrum. Indeed, effective potentials with the same $\Delta$ have different heights, depths and steepnesses, all of which influence effects on the power spectrum. Even for small $\Delta$, the correction can be large depending on the values of $\alpha$ and $\beta$. Therefore, it can hardly be considered a perturbation.

¿From Fig. 4, we can note that for the smaller value of $z$ one obtains a minimum for $C_{\alpha \beta}$ at $\Delta>0$ (which corresponds to a finite value of $\beta$ for a fixed $z$ ). Besides we have no correction $\left(C_{\alpha \beta}=0\right)$ for particular values of $z$ and $\Delta$. This means that we could still have no correction in spite of finite $\alpha$ and $\beta$. In other words,

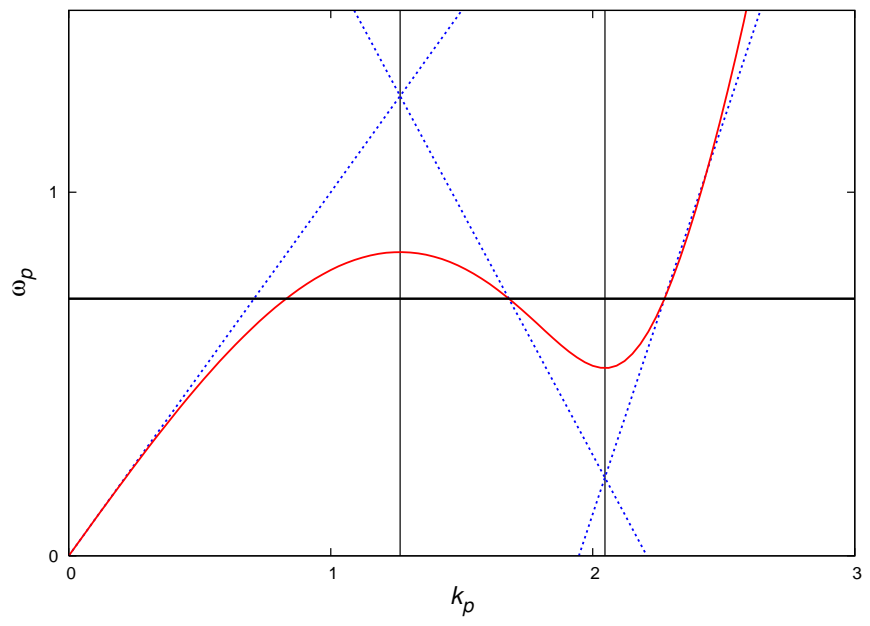

FIG. 5: Three-piecewise linear approximation to the nonlinear d.r. as suggested in Ref. [4]. The horizontal line is $\sqrt{2} H$. Regions 1,2,3 are defined by the vertical lines and labeled from right to left. $k_{p}$ is in units of $M_{p l}$.

this nonlinear d.r. yields no correction whatsoever to the power spectrum if the parameters happen to be around those values.

\section{LINEAR APPROXIMATION}

Since the WKB factor (18) is not much smaller than 1 except for $k \gg a H$, one cannot use the WKB expression (17) for the solution of (24) (except in the forementioned region, of course). Nevertheless, there is indeed an exact solution of Eq. (24) if we approximate the nonlinear dispersion relation by a straight lines, as done in Ref. [4].

If we write $\omega_{i}\left(k_{p}\right)=A_{i} k_{p}+B_{i}$ for each of the regions $i=1,2,3$ (see Fig. [5), the solutions are

$$
\begin{aligned}
\mu_{i}(\eta) & =c_{i} W_{M}\left[\frac{i B_{i}}{H}, \sqrt{\frac{9}{4}-\gamma_{i}}, 2 i A_{i} k \eta\right]+ \\
& +d_{i} W_{W}\left[\frac{i B_{i}}{H}, \sqrt{\frac{9}{4}-\gamma_{i}}, 2 i A_{i} k \eta\right]
\end{aligned}
$$

where $\gamma_{i} \equiv\left(B_{i} / H\right)^{2}$ and $W_{M, W}(\cdot, \cdot, \cdot)$ are Whittaker functions [17]. We pick $A_{i}$ and $B_{i}$ such that the positions of the maximum and minimum of the piecewise d.r. coincide with those of the full nonlinear case and that the first and the second "turning points" $k_{p 1}$ and $k_{p 2}$ are the same as well (see Fig. 5). In this way we have the same $\Delta$ of the exact nonlinear d.r.. Of course, we pick $A_{3}=1$ and $B_{3}=0$, corresponding to the linear d.r. in the long-wavelength (small $k_{p}$ ) limit.

The coefficients $c_{1}, d_{1}$ are given by matching the asymptotic behavior of the previous equation to the initial conditions yielded by the WKB approximation (see 
below). The former is given by [17]

$$
\begin{aligned}
\mu_{1}(\eta) & \approx \mathrm{e}^{-i A_{1} k \eta}\left(2 i A_{1} k \eta\right)^{+i \frac{B_{1}}{H}}\left[c_{1} \frac{\Gamma(1+2 \nu)}{\Gamma\left(\frac{1}{2}+\nu+\kappa\right)}+d_{1}\right]+ \\
& +\mathrm{e}^{+i A_{1} k \eta}\left(2 i A_{1} k \eta\right)^{-i \frac{B_{1}}{H}}\left[c_{1} \frac{\Gamma(1+2 \nu)}{\Gamma\left(\frac{1}{2}+\nu-\kappa\right)}\right]
\end{aligned}
$$

where $\nu \equiv \sqrt{9 / 4-\gamma_{1}}$ and $\kappa \equiv-2 B_{1} / H$. Comparing Eq. (34) to the expected WKB solution

$$
\begin{aligned}
\mu_{w k b}(\eta) & =\frac{1}{\sqrt{2 \omega}} \exp \left[-i \int_{\eta_{i}}^{\eta} \omega(\eta) d \tau\right] \\
& =\frac{1}{\sqrt{2 A_{1} k}}\left(\frac{\eta}{\eta_{i}}\right)^{i \frac{B_{1}}{H}} \mathrm{e}^{-i A_{1} k\left(\eta-\eta_{i}\right)}
\end{aligned}
$$

we get

$$
\left\{\begin{array}{l}
c_{1}=0 \\
d_{1}=\frac{1}{\sqrt{2 A_{1} k}}\left(2 i A_{1} k \eta_{i}\right)^{-i B_{1} / H} .
\end{array}\right.
$$

Note that the expression for $d_{1}$ reduces to the expected form when $A_{1}=1$ and $B_{1}=0$, which correspond to the usual d.r..

The amplitude of the growing mode can be calculated as is usually done in QM. Since we know the exact solution in each region (Eq. (33)), all we have to do is to match them and their derivatives at the boundaries. In matrix notation, at the boundary between regions 1 and 2, we write:

$$
\begin{aligned}
& \left(\begin{array}{ll}
W_{M}^{(1)}\left(\eta_{12}\right) & W_{W}^{(1)}\left(\eta_{12}\right) \\
W_{M}^{\prime(1)}\left(\eta_{12}\right) & W_{W}^{\prime(1)}\left(\eta_{12}\right)
\end{array}\right)\left(\begin{array}{l}
c_{1} \\
d_{1}
\end{array}\right)= \\
= & \left(\begin{array}{ll}
W_{M}^{(2)}\left(\eta_{12}\right) & W_{W}^{(2)}\left(\eta_{12}\right) \\
W_{M}^{\prime(2)}\left(\eta_{12}\right) & W_{W}^{\prime(2)}\left(\eta_{12}\right)
\end{array}\right)\left(\begin{array}{c}
c_{2} \\
d_{2}
\end{array}\right)
\end{aligned}
$$

where the superscripts $(1,2)$ indicate the value of the subscript $i$ in Eq. (33), $\eta_{12} \equiv-k_{p 12} /(H k)$ is the value of $\eta$ at that boundary (see Fig. (5) and (') indicates derivative with respect to $\eta$. We will write the above equation in a more compact form as

$$
\mathcal{M}_{1} \cdot \mathcal{C}_{1}=\mathcal{M}_{2} \cdot \mathcal{C}_{2}
$$

with an obvious notation. Analogously, we can write the matching at the boundary of regions 2 and 3 as

$$
\begin{aligned}
& \left(\begin{array}{cc}
W_{M}^{(2)}\left(\eta_{23}\right) & W_{W}^{(2)}\left(\eta_{23}\right) \\
W_{M}^{\prime(2)}\left(\eta_{23}\right) & W_{W}^{\prime(2)}\left(\eta_{23}\right)
\end{array}\right)\left(\begin{array}{c}
c_{2} \\
d_{2}
\end{array}\right)= \\
= & \left(\begin{array}{cc}
W_{M}^{(3)}\left(\eta_{23}\right) & W_{W}^{(3)}\left(\eta_{23}\right) \\
W_{M}^{\prime(3)}\left(\eta_{23}\right) & W_{W}^{\prime(3)}\left(\eta_{23}\right)
\end{array}\right)\left(\begin{array}{c}
c_{3} \\
d_{3}
\end{array}\right)
\end{aligned}
$$

and as

$$
\mathcal{M}_{3} \cdot \mathcal{C}_{2}=\mathcal{M}_{4} \cdot \mathcal{C}_{3}
$$

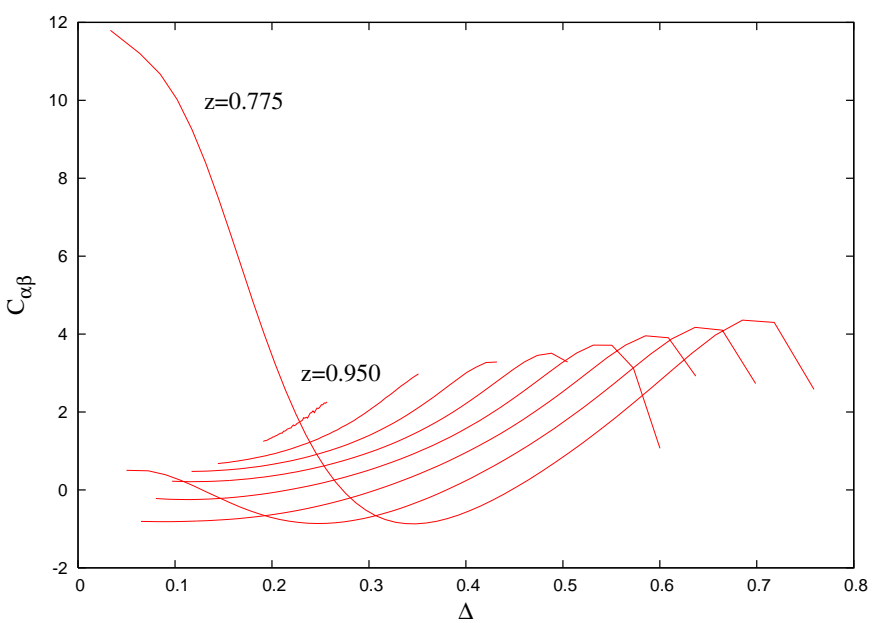

FIG. 6: Correction to the spectrum using the 3-piecewise approximation, with $z$ varying from 0.775 up to 0.950 with step 0.025 , as a function of $\Delta$.

Such a compact notation allow us to write $\mathcal{C}_{3}$ in terms of $\mathcal{C}_{1}$ as

$$
\mathcal{C}_{3}=\mathcal{M}_{4}^{-1} \cdot \mathcal{M}_{3} \cdot \mathcal{M}_{2}^{-1} \cdot \mathcal{M}_{1} \cdot \mathcal{C}_{1}
$$

The coefficient of the growing mode is given by $d_{3}$ the second component of $\mathcal{C}_{3}$ - since it is the coefficient of the divergent Whittaker function when $\eta \rightarrow 0^{-}$:

$$
W_{W}\left(-\frac{i B_{3}}{2 H}, \frac{\sqrt{1-4 \gamma_{3}}}{2}, 2 i A_{3} k^{2} \eta\right) \rightarrow \frac{-i}{k \eta}
$$

while $W_{M} \rightarrow 0$ at the same limit. Therefore,

$$
\mu_{3}(\eta) \rightarrow \frac{-i}{k \eta} d_{3}
$$

as $\eta \rightarrow 0^{-}$. We can thus write the power spectrum as

$$
\begin{aligned}
P_{\phi}(k) & =\frac{k^{3}}{2 \pi^{2}}\left|\frac{\mu_{3}}{a}\right|^{2} \\
& \simeq \frac{H^{2} k}{2 \pi^{2}}\left|d_{3}\right|^{2},
\end{aligned}
$$

in the limit $\eta \rightarrow 0^{-}(k \ll a H)$. This is, as expected, scale invariant.

In Fig. 6 we have plotted the correction of the spectrum, due to this three-piecewise approximation, as a function of $\Delta$. The reason for such large values is that the nonlinear d.r. can be qualitatively different from the linear one $(\omega \sim k)$ at large $|\eta|$ even for small $\Delta$.

As it is clear, comparing Fig. 6 to Fig. 4 the results obtained with this three-piecewise approximation are pretty different from the numerical one obtained with the exact nonlinear d.r..

\section{SEMIANALYTICAL APPROACH}

In this section we use $k_{p}$ as the independent variable. Since it is proportional to $\eta\left(k_{p}=k / a=-\eta H k\right)$, there 
is no particular advantage to choose either way, but this variable is more "physical" and thus one can rely on her/his physical intuition. For a linear d.r., Eq. (12) is then written as

$$
\frac{d^{2} \mu_{k}}{d k_{p}^{2}}+\left(\frac{1}{H^{2}}-\frac{2}{k_{p}^{2}}\right) \mu_{k}=0 .
$$

Using a nonlinear d.r. amounts to the substitution

$$
\frac{1}{H^{2}} \rightarrow \frac{1}{H^{2}} \frac{\omega_{p}^{2}\left(k_{p}\right)}{k_{p}^{2}}
$$

in the previous equation. Although there is no exact solution of Eq. (45) with the substitution (46) when one uses the d.r. (19), we still can get a fair analytical approximation by writing

$$
\begin{aligned}
& \Sigma^{2}\left(k_{p}\right) \equiv \frac{1}{H^{2}} \frac{\omega_{p}^{2}\left(k_{p}\right)}{k_{p}^{2}}-\frac{2}{k_{p}^{2}} \\
& =\frac{\beta}{H^{2}} k_{p}^{4}-\frac{\alpha}{H^{2}} k_{p}^{2}-\frac{2}{k_{p}^{2}}+\frac{1}{H^{2}} \\
& \approx\left\{\begin{array}{c}
\Sigma_{1}^{2}\left(k_{p}\right) \equiv \frac{\beta}{H^{2}} k_{p}^{4}-\frac{\alpha}{H^{2}} k_{p}^{2}+\frac{1}{H^{2}}-c, k_{p} \geq k_{*} \\
\Sigma_{2}^{2}\left(k_{p}\right) \equiv-\frac{\alpha}{H^{2}} k_{p}^{2}+\frac{1}{H^{2}}-\frac{2}{k_{p}^{2}}-d \quad, k_{p} \leq k_{*}
\end{array}\right.
\end{aligned}
$$

where $c, d$ and the matching point $k_{*}$ are defined by requiring that

$$
\left\{\begin{array}{l}
\Sigma_{1}^{2}\left(k_{*}\right)=\Sigma_{2}^{2}\left(k_{*}\right)=\Sigma^{2}\left(k_{*}\right) \\
\left.\frac{d \Sigma_{1}^{2}}{d k}\right|_{k_{*}}=\left.\frac{d \Sigma_{2}^{2}}{d k}\right|_{k_{*}}
\end{array},\right.
$$

which correspond to $k_{*}=\left(H^{2} / \beta\right)^{1 / 6}, d=-\left(\beta / H^{2}\right)^{1 / 3}$ and $c=2\left(\beta / H^{2}\right)^{1 / 3}$. The approximated expressions have the same limiting behaviors (both at $k_{p} \rightarrow 0$ and $k_{p} \rightarrow$ $\infty)$ than the original one. That feature provides another piece of information on the dependence of the outcome on the details of the nonlinear d.r. for intermediate $k_{p}$, as opposed to its small- and large-scale limits. See Fig. 7 for a qualitative comparison.

The calculation itself is carried out by the usual approach in $\mathrm{QM}$, as in the previous section. The requirements that the "wave function" and its first derivative are continuous at $k_{*}$ are easily accomplished since the solutions of the equation of motion (45146) with the approximations (47) are known analytically. For $k>k_{*}$ we find:

$$
\begin{gathered}
\frac{d^{2} f_{1}\left(k_{p}\right)}{d k_{p}^{2}}-\Sigma_{1}^{2}\left(k_{p}\right) f_{1}\left(k_{p}\right)=0 \\
f_{1}\left(k_{p}\right)=c_{1} \exp \left[+\frac{i}{H}\left(-\frac{\alpha}{2 \sqrt{\beta}} k_{p}+\frac{\sqrt{\beta}}{3} k_{p}^{3}\right)\right] H t_{1}\left(k_{p}\right)+ \\
+d_{1} \exp \left[-\frac{i}{H}\left(-\frac{\alpha}{2 \sqrt{\beta}} k_{p}+\frac{\sqrt{\beta}}{3} k_{p}^{3}\right)\right] H t_{2}\left(k_{p}\right)
\end{gathered}
$$

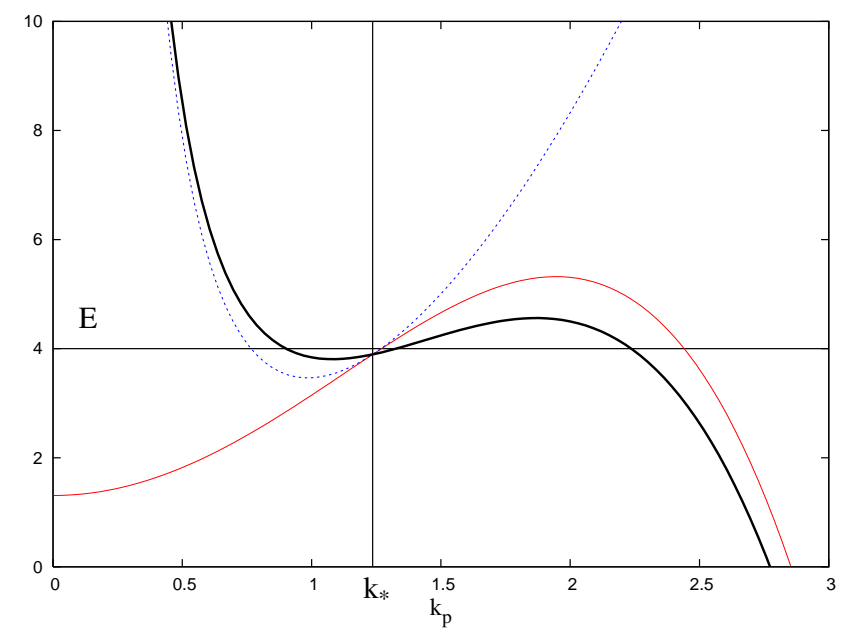

FIG. 7: Effective potential $V_{\text {eff }}\left(k_{p}\right)$ (thick black line) and its approximations (thin colored lines) given by $V_{\text {eff }}\left(k_{p}\right)=E-$ $\Sigma^{2}\left(k_{p}\right)$ and Eqs. (48), for $\bar{\alpha}=0.1325, \bar{\beta}=0.004375$ and $H=0.5 M_{p l}$, as a function of $k_{p}$ (in $M_{p l}$ units). The straight horizontal line is $E \equiv 1 / H^{2}$. The vertical line marks the value of $k_{*}$ (see text for definition).

where $H t_{1}\left(k_{p}\right) \equiv \operatorname{HeunT}(A, 0, B,+i \zeta) \quad$ and $H t_{2}\left(k_{p}\right) \equiv \operatorname{HeunT}(A, 0, B,-i \zeta)$ are triconfluent Heun functions [18], with

$$
\begin{aligned}
A & \equiv\left(\frac{3}{2 H^{2} \sqrt{\beta}}\right)^{2 / 3}\left(-1+c H^{2}+\frac{\alpha^{2}}{4 \beta}\right) \\
B & \equiv\left(\frac{3}{2 H^{2} \beta^{2}}\right)^{1 / 3} \alpha \\
\zeta & \equiv\left(3 \sqrt{2} \beta^{1 / 4} H\right)^{1 / 3} k_{p} .
\end{aligned}
$$

We can constrain the coefficients $\left(c_{1}, d_{1}\right)$ in Eq. (50) by comparing the asymptotic behavior of the above equation to the expected WKB one, Eq. (26). The asymptotic limit of interest here is taken along a Stokes line $(\arg (i \zeta)=\pi / 2)$ of the triconfluent Heun function, which means that there are two equally dominant terms in the asymptotic expansion:

$$
\operatorname{HeunT}(A, 0, B, i \zeta) \sim \zeta^{-1}\left[a+b \exp \left(-i \zeta^{3}\right)\right]
$$

as $\zeta \rightarrow+\infty$, where $a$ and $b$ are constants. Since neither one is dominant over the other, both must be taken into account. The forementioned comparison yields

$$
\left\{\begin{array}{l}
d_{1} b-c_{1} a=0 \\
-c_{1} b+d_{1} a=C
\end{array}\right.
$$

where $C \equiv i /\left[\left(3 H \sqrt{2} \beta^{1 / 4}\right)^{1 / 3} \sqrt{k}\right]$. There are only 2 equations for 4 unknowns, which should be expected from the lack of a dominant behavior in the asymptotic expansion along a Stokes line, as mentioned above. One could choose whichever 2 of the above parameters (say, $a$ and $b)$ to be specified by comparing the final result to the 
outcome of the numerical calculation using the approximated effective potential. Formally, that determination is supposed to be done at every value of $\alpha$ and $\beta$, which would render our semianalytical approach useless. Nevertheless, we have found that writing $b$ and $d_{1}$ in terms of $a$ and $c_{1}$ yields a qualitatively good behavior for different values of $z$, i.e, for different pairs $\{\alpha, \beta\}$ when compared to the numerical calculation (see Fig. 8 below). Such procedure yields

$$
\begin{aligned}
b & =\frac{c_{1}}{d_{1}} a \\
d_{1} & =\frac{-C \pm \sqrt{C^{2}+4 a^{2} c_{1}^{2}}}{2 a}
\end{aligned}
$$

The sign in the above equation was again numerically determined to be the lower one (see below). Following this line of reasoning, we used $a=0.1$ and $c_{1}=-1.8$ for all $z$.

The last step of the semianalytical procedure is the evolution in the second region, when $k<k_{*}$ :

$$
\frac{d^{2} f_{2}\left(k_{p}\right)}{d k_{p}^{2}}-\Sigma_{2}^{2}\left(k_{p}\right) f_{1}\left(k_{p}\right)=0
$$

whose exact solution is

$$
\begin{aligned}
f_{2}\left(k_{p}\right) & =c_{2} \frac{1}{\sqrt{k_{p}}} W_{M}\left(D, \frac{3}{4}, \frac{\sqrt{\alpha} k_{p}^{2}}{H}\right)+ \\
& +d_{2} \frac{1}{\sqrt{k_{p}}} W_{W}\left(D, \frac{3}{4}, \frac{\sqrt{\alpha} k_{p}^{2}}{H}\right)
\end{aligned}
$$

where $D \equiv\left(1-d H^{2}\right) /(4 H \sqrt{\alpha})$.

The coefficient $d_{2}$ determines the amplitude of the perturbations since the function $W_{W}\left(k_{p}\right)$ is the growing solution:

$$
\lim _{k_{p} \rightarrow 0} \frac{W_{W}\left(k_{p}\right)}{\sqrt{k_{p}}}=\frac{\sqrt{\pi} H^{1 / 4}}{2 \alpha^{1 / 8} \Gamma(\xi)} \frac{1}{k_{p}}
$$

where $\xi \equiv\left(\frac{5}{4}-\frac{1-d H^{2}}{4 H \sqrt{\alpha}}\right)$. The above expression allows us to write the spectrum as

$$
\begin{aligned}
P_{\phi}(k) & \equiv \frac{k^{3}}{2 \pi^{2}}\left|\frac{f_{2}}{a}\right|^{2} \\
& \simeq \frac{k \sqrt{H}}{8 \pi \alpha^{1 / 4} \Gamma^{2}(\xi)}\left|d_{2}\right|^{2} \\
& =\left(\frac{H}{2 \pi}\right)^{2}\left(1+C_{\alpha \beta}\right),
\end{aligned}
$$

in the superhorizon limit $k_{p} \ll H$. The coefficient $d_{2}$ is determined by the forementioned procedure, requiring that the function and its derivative are continuous at $k=k_{*}$. In matrix notation, it can be written as

$$
\left(\begin{array}{cc}
g_{1}\left(k_{*}\right) & h_{1}\left(k_{*}\right) \\
\left(\frac{d g_{1}\left(k_{p}\right)}{d k_{p}}\right)_{k_{p}=k_{*}} & \left(\frac{d h_{1}\left(k_{p}\right)}{d k_{p}}\right)_{k_{p}=k_{*}}
\end{array}\right)\left(\begin{array}{c}
c_{1} \\
d_{1}
\end{array}\right)=
$$

$$
=\left(\begin{array}{cc}
g_{2}\left(k_{*}\right) & h_{2}\left(k_{*}\right) \\
\left(\frac{d g_{2}\left(k_{p}\right)}{d k_{p}}\right)_{k_{p}=k_{*}} & \left(\frac{d h_{2}\left(k_{p}\right)}{d k_{p}}\right)_{k_{p}=k_{*}}
\end{array}\right)\left(\begin{array}{c}
c_{2} \\
d_{2}
\end{array}\right)(64)
$$

where

$$
\begin{aligned}
g_{1}\left(k_{p}\right) & \equiv \exp \left[\frac{+i}{H}\left(\frac{-\alpha}{2 \sqrt{\beta}} k_{p}+\frac{\sqrt{\beta}}{3} k_{p}^{3}\right)\right] H t_{1}\left(k_{p}\right) \\
h_{1}\left(k_{p}\right) & \equiv \exp \left[\frac{-i}{H}\left(\frac{-\alpha}{2 \sqrt{\beta}} k_{p}+\frac{\sqrt{\beta}}{3} k_{p}^{3}\right)\right] H t_{2}\left(k_{p}\right) \\
g_{2}\left(k_{p}\right) & \equiv \frac{1}{\sqrt{k_{p}}} W_{M}\left(D, \frac{3}{4}, \frac{\sqrt{\alpha} k_{p}^{2}}{H}\right) \\
h_{2}\left(k_{p}\right) & \equiv \frac{1}{\sqrt{k_{p}}} W_{W}\left(D, \frac{3}{4}, \frac{\sqrt{\alpha} k_{p}^{2}}{H}\right)
\end{aligned}
$$

which can be written in a more compact form, as in the previous section, as

$$
\mathcal{M}_{1} \cdot \mathcal{C}_{1}=\mathcal{M}_{2} \cdot \mathcal{C}_{2}
$$

As before, one can invert such equation and write

$$
\mathcal{C}_{2}=\mathcal{M}_{2}^{-1} \cdot \mathcal{M}_{1} \cdot \mathcal{C}_{1}
$$

which shows once more that $d_{2}$ is a linear combination of $c_{1}$ and $d_{1}$. Since we have fixed $c_{1}$ from the beginning, we are not able to find analytically the dependence of $d_{2}$ on $k$ and, therefore, to say if the spectrum is scale invariant, as it should be. Nevertheless, we approach the same problem numerically and we verify that the problem exhibits such invariance.

In order to be able to measure the acuracy of the semianalytical approach, we have used the approximation (47) and numerically evolved the initial conditions (28, 29). The evolution was split in two pieces: for $k>k_{*}$ and $k<k_{*}$. Such calculation, besides fixing the sign in Eq. (57), also yields the "best" (i.e., more robust with respect to changes in $\alpha$ and $\beta$ ) values of $c_{1}=-1.8$ and $a=0.1$.

In Fig. 8 we show the function $C_{\alpha \beta}$ for different values of $z$ as a function of $\Delta$, as given by the numerical evolution using the approximated expressions for the effective potential and as given by the semianalytical approach with the same approximation. We can see that the correction depends also on $z$, showing that $\Delta$ is not the only variable to work with.

The semianalytical solution is not expected to work well for small $\bar{\beta}$, which means, for a fixed $z$, small $\Delta$. For that, we should have taken into account the next-toleading order terms in the asymptotic expansion of the Heun functions, Eq. (54). That would have, however, introduced new parameters that would have had to be fixed by comparing to the numerical solution once more. Since we see no advantage in having a large number of such parameters, we did not do so.

We note that we reproduce a minimum $C_{\alpha \beta}$ at a finite $\Delta$ as in the previous section. This approximation yields a 


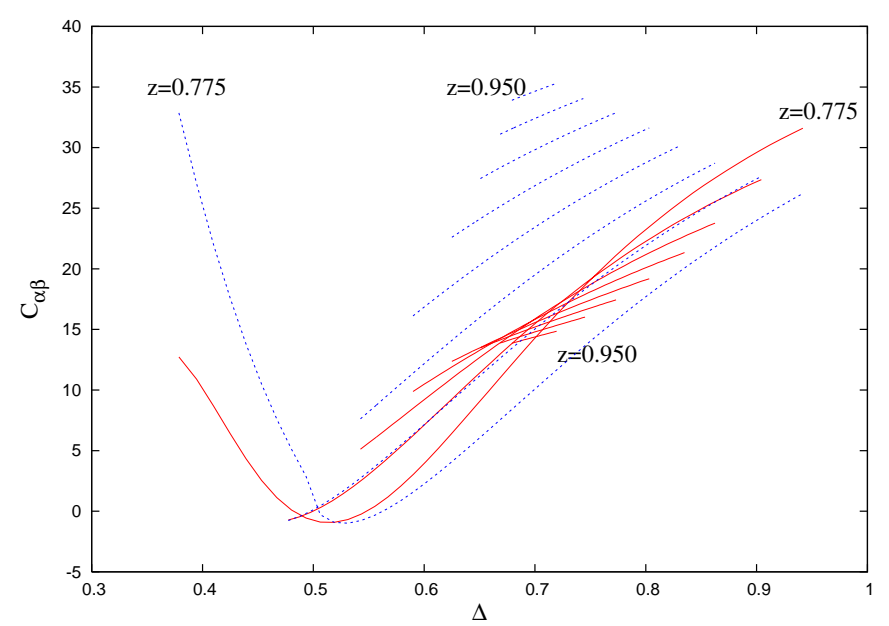

FIG. 8: Correction $C_{\alpha \beta}$ as a function of $\Delta$ when $z$ varies from 0.775 to 0.950 (as shown by the labels) for the semianalytical approach (dashed blue lines) and for the numerical evolution with approximated effective potential (47) (full red lines).

correction $C_{\alpha \beta}$ with a good qualitative behavior as compared to Fig. 4, but a large difference in the magnitude of the effect.

\section{POWER-LAW INFLATION}

A numerical approach can also be applied to find the correction to the power spectrum in a realistic model of inflation for the nonlinear dispersion relation under consideration. In this section we restrict ourselves to the case of power-law inflation [19] where, in proper time, $a(t) \sim t^{p}$, with $p>1$. This expansion is generated by an exponential potential

$$
V(\phi)=V_{0} \exp \left[-\frac{\lambda}{M_{p l}}\left(\phi-\phi_{i}\right)\right]
$$

with $V_{0}=\frac{M_{p l}^{2}}{t_{i}^{2}} p(3 p-1)$ and $\lambda=\left(\frac{2}{p}\right)^{1 / 2}$. The scale factor and the homogeneus solution of the scalar field are given by

$$
\begin{aligned}
a(t) & =\left(\frac{t}{t_{i}}\right)^{p}, \\
\phi(t) & =\phi_{i}+M_{p l}(2 p)^{1 / 2} \log \frac{t}{t_{i}}
\end{aligned}
$$

respectively, and the slow-roll parameters by

$$
\epsilon_{1} \equiv \frac{M_{p l}^{2}}{2}\left(\frac{V_{\phi}}{V}\right)^{2}=\frac{1}{p}, \epsilon_{2} \equiv M_{p l}^{2} \frac{V_{\phi \phi}}{V}=\frac{2}{p},
$$

where $V_{\phi} \equiv d V / d \phi$ and so on. In conformal time $\eta$, the scale factor and the Hubble parameter become

$$
a(\eta)=\left(\frac{\eta}{\eta_{i}}\right)^{\frac{p}{1-p}}, H(\eta)=-\frac{p}{p-1}\left(\frac{\eta}{\eta_{i}}\right)^{\frac{p}{p-1}} \frac{1}{\eta}
$$

with $\eta_{i}=t_{i} /(1-p)$. The equation of motion for the Mukhanov variable, Eq.(8), becomes (using $\eta$ as the independent variable)

$$
\mu_{s k}^{\prime \prime}+\left(k^{2}-\frac{a^{\prime \prime}}{a}\right) \mu_{s k}=0
$$

and the canonically normalized solution, associated with the adiabatic vaccum for $k \gg a H$, is given by (see, for example, 20])

$$
\mu_{k}=\left(-\frac{\pi \eta}{4}\right)^{1 / 2} H_{\nu}^{(1)}(-k \eta)
$$

with $\nu=3 / 2+1 /(p-1)$. So, in the long-wavelength limit, we obtain the following scale dependent power spectrum

$$
P_{Q}(k)=\frac{1}{(2 \pi)^{2}}\left(-\frac{2}{\eta_{i}}\right)^{\frac{2 p}{p-1}} \frac{1}{\pi} \Gamma^{2}(\nu) k^{-\frac{2}{p-1}}
$$

with a spectral index $n_{s}=1-\frac{2}{p-1}$. Using this result, namely the dependence on $\mathrm{k}$ of the power spectrum, the range $p<60$ is disfavored at $2 \sigma$ by the observation (see, for example, [21]).

Now we want to see if the introduction of the particular nonlinear d.r. given in Eq. (19) changes the dependence of the power spectrum on $k$. Let us set our background: Eq. (76) can be written, using Eq. (75), as

$$
\mu_{k}^{\prime \prime}+\left[k^{2}-\frac{2 p^{2}-p}{(1-p)^{2}} \frac{1}{\eta^{2}}\right] \mu_{k}=0 .
$$

For $p \gg 1$, this can be approximated as

$$
\mu_{k}^{\prime \prime}+\left[k^{2}-2 a^{2} H^{2}\right] \mu_{k}=0,
$$

so, in this limit and following the same reasoning as before, to obtain a positive nonlinear d.r. that gives three real distinct classical turning-points $\left(\omega_{p}^{2}=2 H^{2}\right)$ at the initial time [25] we require that

$$
3 / 4<z<1 \quad, \quad \frac{g(z)}{H\left(\eta_{i}\right)^{2}}<\alpha<\frac{f(z)}{H\left(\eta_{i}\right)^{2}} .
$$

with $g(z)$ and $f(z)$ given in Eqs. (22]23).

The new equation of motion will be given by

$$
\mu_{k}^{\prime \prime}+\left[a^{2}\left(\frac{k^{2}}{a^{2}}-\alpha \frac{k^{4}}{a^{4}}+\beta \frac{k^{6}}{a^{6}}\right)-\frac{2 p^{2}-p}{(1-p)^{2}} \frac{1}{\eta^{2}}\right] \mu_{k}=0
$$

and, repeating the calculation in Sec. [V] with the new background, one obtains the following initial conditions

$$
\begin{aligned}
& \mu_{w k b}\left(\eta_{i}\right)=\frac{1}{\sqrt{2} \beta^{1 / 4} k^{3 / 2}} \\
& \mu_{w k b}^{\prime}\left(\eta_{i}\right)=-\frac{1}{\sqrt{2} \beta^{1 / 4} k^{3 / 2}} \frac{p}{p-1} \frac{1}{\eta_{i}}-i \frac{\beta^{1 / 4}}{\sqrt{2}} k^{3 / 2}
\end{aligned}
$$

We now proceed with the numerical analysis taking $t_{i}=(p-1) / M_{p l}$ (and thus $\left.\eta_{i}=-1 / M_{p l}\right)$. The restriction to apply the adiabatic initial condition $\left(-k \eta_{i} \gg 1\right)$ 


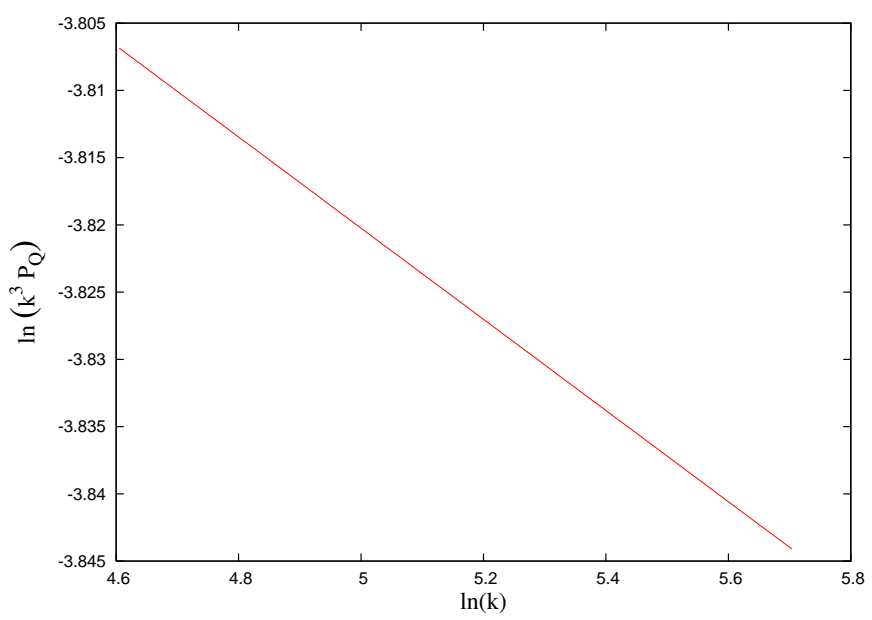

FIG. 9: Logarithm of the power spectrum in a power-law model of inflation in function of $\ln (k)$ for a linear d.r.. $k$ is in units of $M_{p l}$.

becomes equivalent to $k \gg M_{p l}$; therefore, we are indeed in the energy scale of TPP. Besides, with those initial conditions and in the limit $p \gg 1$, we have $H\left(\eta_{i}\right) \simeq M_{p l}$ and the new d.r. becomes very different from the linear one only for $k=\mathcal{O}\left(M_{p l}\right)$. For the numerical analysis we take $z=0.80, \alpha=\frac{f(0.80)+g(0.80)}{2 H\left(\eta_{i}\right)^{2}}$ and the limiting value $p=60$, making the analysis for $k$ between $100 M_{p l}$ and $300 M_{p l}$ and going from $\eta_{i}$ to a time for which $k \ll a H$. We give in Fig. 9 the logarithm of the power spectrum calculated for the standard linear case in function of $\ln (k)$, while in Fig. 10 the logarithm of the power spectrum calculated for the nonlinear d.r. case in function of $\ln (k)$ for many different points inside the aforementioned range of $k$. As one can see from this plot, the power spectrum for the nonlinear case still has a power-law dependence on $k$, but this d.r. produces a change both in the normalization factor and in the spectral index when compared to the linear case. Fitting the data in Fig. 10 we obtain a spectral index smaller than that of the linear case: 0.857 instead of 0.966 . The new spectral index would be almost the same as that of the linear case if we have taken $p \sim 15$. One obtains similar results starting from different values of $p$ or with different values for the parameters $\alpha$ and $\beta$. Therefore, the introduction of the nonlinear d.r. gives a stronger dependence of the power spectrum on $k$ and, as a consequence, a possible different range of disfavored values for $p$.

A different approach to this problem in a power-law model is shown in 22]. The authors use the minimumuncertainty principle to fix the initial conditions and also find corrections to the power spectrum. We also would like to underline that our results are not in disagreement with Ref. [23]. Indeed, we consider a regime for which $H$ is of the order of $M_{p l}$ while the results of [23] assume $H \ll M_{p l}$.

Using the same parameters as in Fig. 10, we have plotted in Fig. 11 the behavior of $1 /(1+W)$, where $W$ is

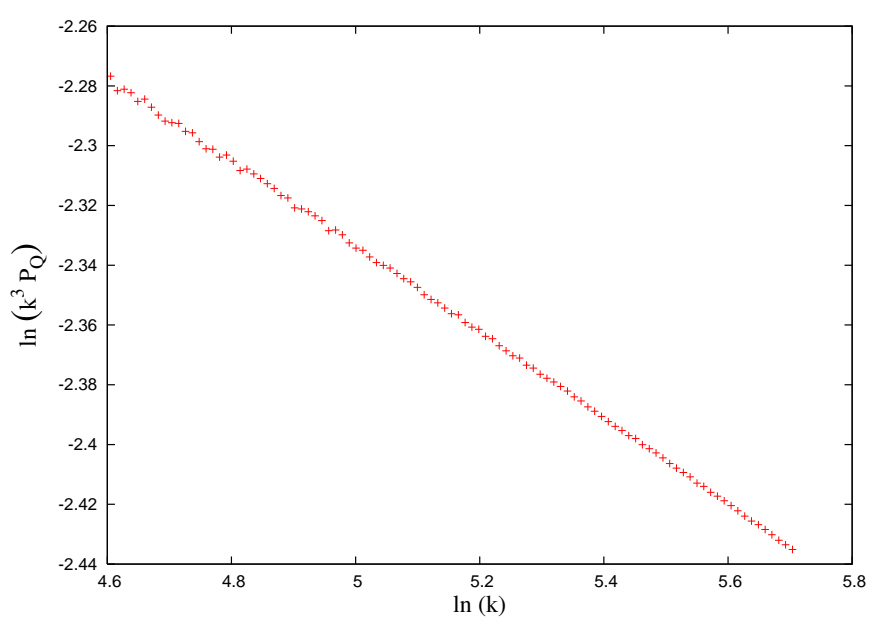

FIG. 10: Logarithm of the power spectrum in a power-law model of inflation using the nonlinear d.r. (12) in function of $\ln (k) . k$ is in units of $M_{p l}$.

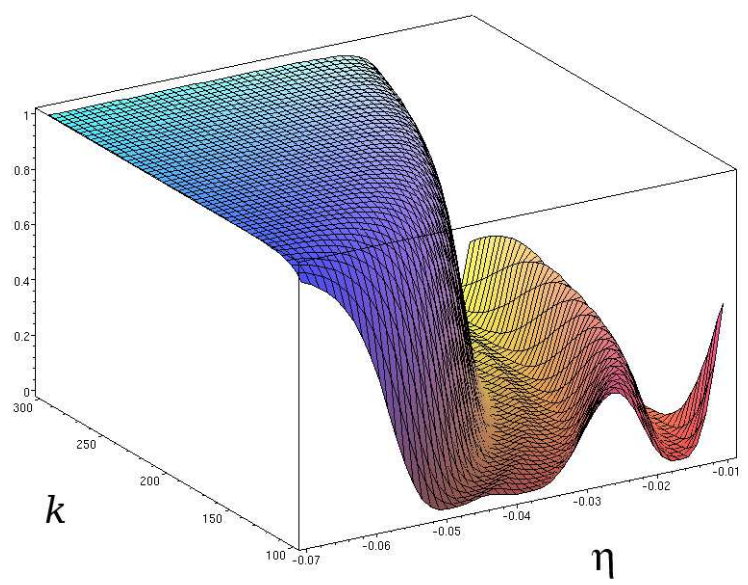

FIG. 11: Plot of the function $1 /(1+W)$ in terms of the wavenumber $k$ (in units of $M_{p l}$ ) and the conformal time $\eta$ using the same parameters as in Fig. 10 (see text for the precise values). One can clearly see that the WKB approximation holds $(W \ll 1)$ only for early times.

defined in Eq. (18). One can easily see that the WKB approximation does not hold at small absolute values of the conformal time, which stresses our very point [26].

\section{CONCLUSIONS}

In this paper we have shown that the nonlinear d.r. (19) presents nonlinear results, i.e, which are not shown in the perturbative approaches used in previous papers in the subject. In particular, the correction $C_{\alpha \beta}$ can be almost as large as 10 depending on the value of the parameters $\alpha, \beta$. Therefore, it can hardly be considered a perturbation.

Our results agree, in part, with the literature on the 
effects on the power spectrum obtained using different approachs to mimic TPP. In fact, also using different approachs, the effects are always of the order of $H / M_{p l}$ (see 4] and references cited therein) which in our case is $\mathcal{O}(1)$

We have compared previously used approximations and introduced a new one, which allowed a semianalytical calculation.

The fundamental point behind such a large correction factor lies in the break of the WKB approximation at early (conformal) times. If the d.r. at this moment is highly nonlinear, as it happens here, the corrections are bound to be large [24].

However, from Fig. 4 we can see that for particular value of $z$ and $\Delta$ we obtain no correction to the power spectrum. Namely, that we could still have no correction in spite of finite $\alpha$ and $\beta$. In other words, this nonlinear d.r. yields no correction whatsoever to the power spectrum if the parameters happen to be around those values.

In the last section we have used a power-law model of inflation as the background in order to understand what features were particular to the de Sitter (exponential) expansion. It is clear that one would always get a scale-free spectrum in a de Sitter background, but what would happen to the spectral index $n_{s}$ in a different one? We have shown that this particular d.r. (19) yields a even smaller $n_{s}$ as compared to power-law inflation with a linear d.r., and therefore, it is strongly disfavored by observational results. On the other hand, this result suggests that another nonlinear d.r. may yield also a different correction (perhaps in the opposite direction) to the value of $n_{s}$ in a power-law background.

We have not addressed the question on back-reactions which may play an important role, since the corrections to the power spectrum are not perturbative ones. Such implications are beyond the scope of this work and deserve a separate study, which will be published elsewhere.

Acknowledgments: We would like to thank R. Ansari, A. Sarkar and M. Schulze for useful discussions and Professor R.H. Brandenberger for useful correspondence. S.E.J. thanks Professor Duval for her help on the Heun functions and acknowledges financial support from ICTP and from CNPq.
[1] R.H. Brandenberger, hep-ph/9910410.

[2] J. Martin and R.H. Brandenberger, Phys. Rev. D 63, 123501 (2001); R.H. Brandenberger and J. Martin, Mod. Phys. Lett. A 16, 999 (2001).

[3] M. Lemoine, M. Lubo, J. Martin and J.-P. Uzan, Phys. Rev. D 65, 023510 (2001).

[4] J. Martin and R. Brandenberger, Phys. Rev. D 68, 063513 (2003).

[5] L. P. Grishchuk, Zh. Eksp. Teor. Fiz. 67, 825 (1974) [Sov. Phys. JETP 40, 409 (1975)].

[6] V. F. Mukhanov, Zh. Eksp. Teor. Fiz. 94,No. 7, 1 (1988) [Sov. Phys. JETP 67, 1297 (1988)].

[7] Eugen Merzbacher, "Quantum Mechanics" (John Wiley \& Sons, 1970), $3^{\text {rd }}$ edition.

[8] J. Martin and D. J. Schwarz, Phys. Rev. D 67, 083512 (2003)

[9] W.G. Unruh, Phys. Rev D 51, 2827 (1995).

[10] S. Corley and T. Jacobson, Phys. Rev. D 54, 1568 (1996).

[11] S. Cremonini, Phys. Rev. D 68, 063514 (2003); J. Kowalski-Glikman, Phys. Lett. B 499, 1 (2001); H.-C. Kim, J. H. Yee and C. Rim, Phys. Rev. D 72, 103523 (2005).

[12] A.A. Abrikosov, "Methods of Quantum Field Theory in Statistical Physics" (Dover Pub., 1975); T. Jacobson, Phys. Rev. D 44, 1731 (1991).

[13] Robert C. Myers and M. Pospelov, Phys. Rev. Lett. 90, 211601 (2003).

[14] R.H. Brandenberger and J. Martin, Phys. Rev. D 71,
023504 (2005).

[15] U. H. Danielsson, Phys. Rev. D 66, 023511 (2002).

[16] http://www.gnu.org/software/gsl

[17] M. Abramowitz and I.A. Stegun, "Handbook of mathematical functions with formulas, graphs, and mathematical tables" (Washington, D.C.: National Bureau of Standards, 1964).

[18] A. Ronveaux (Ed.), "Heun's differential equations" (Oxford University Press, 1995).

[19] F. Lucchin and S. Matarrese, Phys. Rev. D 32, 1316 (1985); D. Lyth and E. Stewart, Phys. Lett. B 274, 168 (1992).

[20] G. Marozzi, Phys. Rev. D 76, 043504 (2007).

[21] F. Finelli, M. Rianna and N. Mandolesi, J. Cosmol. Astropart. Phys. 12 (2006) 006.

[22] G.L. Alberghi, R. Casadio and A. Tronconi, Phys. Lett. B 579, 1 (2004).

[23] A. A. Starobinsky, JETP Lett. 73, 371 (2001).

[24] J.C. Niemeyer and R. Parentani, Phys. Rev. D 64, 101301(R) (2001).

[25] The Hubble factor is time-dependent but for $p \gg 1$ it becomes almost constant (see Eq.(75)).

[26] Although WKB is not a good approximation, we have calculated the spectral index with the nonlinear d.r. using, in a strict and forward way, the WKB solutions (17) and the same parameters as before, for the sake of comparison. The spectrum turns out to be strongly (blue) shifted from the correct (numerical) value: $n_{s}^{\mathrm{wkb}}=1.22$. 